\title{
Determination of the mixing angle between new charmonium states
}

\author{
T. M. Aliev ${ }^{1,2, a}$, M. Savc1 ${ }^{1, b}$ \\ ${ }^{1}$ Physics Department, Middle East Technical University, 06800 Ankara, Turkey \\ ${ }^{2}$ Institute of Physics, Baku, Azerbaijan
}

Received: 12 February 2015 / Accepted: 31 March 2015 / Published online: 28 April 2015

(C) The Author(s) 2015. This article is published with open access at Springerlink.com

\begin{abstract}
Using the pictures for $X(3872)$ as a mixture of charmonium and molecular $D^{* 0} D^{0}$ states; $Y(3940)$ as a mixture of $\chi_{c 0}$ and $D^{*} D^{* *}$ states, and $Y(4260)$ as a mixture of the tetra-quark state with charmonium states, the corresponding mixing angles are estimated within the QCD sum rules. We find that our predictions for the mixing angles of the $X$ (3872), $Y(4260)$, and $Y(3940)$ states are considerably smaller compared to work in which the mixing angles are estimated from the condition in reproducing the mass of these states. Our conclusion is that the considered pictures for the $X(3872)$, $Y(4260)$, and $Y(3940)$ states are not successful in describing these states.
\end{abstract}

\section{Introduction}

The analysis of the spectroscopy and decays of the heavy flavored mesons is an essential source for obtaining useful information about the dynamics of QCD at "low" energies. Remarkable progress in this direction has been made on the experimental side. Starting from the observation of $X(3872)$ [1] up to the present time 23 new charmonium line states have been discovered (for a review and relevant references to the original work on the charmonium spectroscopy, see for example [2-7]). All observed charmonium states might have more complex structures compared to those predicted by the simple quark model. These new states (referred to as $X Y Z$ states in the text) can be potential candidates of exotic states, and for this reason theorists make great efforts for understanding the dynamics of these states [2-7]. There are two attractive pictures in the interpretation of all observed states: tetra-quark, and bound states of two mesons (meson molecules).

\footnotetext{
a e-mail: taliev@metu.edu.tr

b e-mail: savci@metu.edu.tr
}

The theoretical approaches which have been employed in the investigation of these states are QCD sum rules, lattice QCD, effective Lagrangian method, chiral perturbation theory, quark model, etc. Among all approaches the QCD sum rules method occupies a special place [8], which is based on the fundamental QCD Lagrangian. The mass and some of the strong coupling constants of $X Y Z$ mesons with light mesons are widely discussed in the framework of the QCD sum rules method. It is assumed in [9] that $X(3872)$ is a mixture of charmonium and molecular states, whose interpolating current is taken as

$j_{\mu}=\cos \theta j_{\mu}^{(2)}+\sin \theta j_{\mu}^{(4)}$,

where

$j_{\mu}^{(2)}=\frac{1}{6 \sqrt{2}}\langle\bar{q} q\rangle \bar{c} \gamma_{\mu} \gamma_{5} c$,

$j_{\mu}^{(4)}=\frac{1}{\sqrt{2}}\left[\left(\bar{u} \gamma_{5} c\right)\left(\bar{c} \gamma_{\mu} u\right)-\left(\bar{u} \gamma_{\mu} c\right)\left(\bar{c} \gamma_{5} u\right)\right]$,

and whose analysis in the QCD sum rules method predicted that if the mixing angle $\theta$ lies in the range $(9 \pm 4)^{0}$, it can provide good agreement with the experimental value of the mass and decay width. $X(3872)$ as an axial tetra-quark state is analyzed within the QCD sum rules method in [10], and the mass is found to be $m_{X}=3.87 \mathrm{GeV}$.

Using the QCD sum rules, the mass and the decay width of the channel $J / \Psi \omega$ for the $Y(3940)$ state is studied in $[11,12]$, assuming that it is described by the mixed scalar $\chi_{c 0}$ and $D^{*} \bar{D}^{*}$ states, i.e.,

$j=-\frac{\langle\bar{q} q\rangle}{\sqrt{2}} \cos \theta j_{\chi_{c 0}}+\sin \theta j_{D^{*} \bar{D}^{*}}$,

where $j_{\chi_{c 0}}=\bar{c} c$ and $j_{D^{*} \bar{D}^{*}}=\left(\bar{q} \gamma_{\mu} c\right)\left(\bar{c} \gamma^{\mu} q\right)$. As a result of this study it is found that one can reproduce the mass and decay width of $Y$ (3940) in very good agreement with the experimental result if the mixing angle is chosen to be 
$\theta=(76 \pm 5)^{0}$. Being a molecular $D^{*} D^{*}$ state, $Y(3940)$ is analyzed in the framework of the QCD sum rules in [13].

A similar analysis is carried out in [14] for the $Y(4260)$ state by assuming that it can be described by a mixture of the tetra-quark and charmonium currents, i.e.,

$j_{\mu}=\cos \theta j_{\mu}^{(2)}+\sin \theta j_{\mu}^{(4)}$,

where

$$
\begin{aligned}
j_{\mu}^{(4)}= & \frac{1}{\sqrt{2}} \varepsilon_{a b c} \varepsilon_{d e c}\left[\left(q_{a}^{T} C \gamma_{5} c_{b}\right)\left(\bar{q}_{d} \gamma_{\mu} \gamma_{5} C \bar{c}^{T}\right)\right. \\
& \left.+\left(q_{a}^{T} C \gamma_{5} \gamma_{\mu} c_{b}\right)\left(\bar{q}_{d} \gamma_{5} C \bar{c}^{T}\right)\right], \\
j_{\mu}^{(2)}= & \frac{1}{\sqrt{2}}\langle\bar{q} q\rangle \bar{c} \gamma_{\mu} c,
\end{aligned}
$$

and it is found that the experimental result can be reproduced by setting the mixing angle to $\theta=(53 \pm 5)^{0}$.

Note that the flavor mixing in the light quark sector is studied in $[15,16]$. In these works the $U_{A}(1)$ anomaly plays a considerably important role in the flavor mixing, which leads to the mixing among the hadrons, and further enables estimation of the mixing angles for the $\eta-\eta^{\prime}$ and $\omega-\phi$ states.

In determining the mixing angles we shall follow the method presented in $[17,18]$, in which the mixing angles among the hadronic states are determined from the QCD sum rules method. The main idea in the determination of the mixing angles is as follows. If the pure states $H_{1}^{0}$ and $H_{2}^{0}$ do mix, then the physical states with definite mass should be represented as linear combinations of these states. According to the QCD sum rules method, each hadronic state is described by the corresponding interpolating current carrying the same quantum numbers as the relevant hadrons. Therefore, the interpolating currents corresponding to the physical states can be represented as a linear combination of the "bare" currents as follows:

$$
\begin{aligned}
& j_{H_{1}}=\cos \theta j_{H_{1}^{0}}+\sin \theta j_{H_{2}^{0}}, \\
& j_{H_{2}}=-\sin \theta j_{H_{1}^{0}}+\cos \theta j_{H_{2}^{0}},
\end{aligned}
$$

where $j_{H_{1}^{0}}$ and $j_{H_{2}^{0}}$ are the bare currents, and $\theta$ is the mixing angle between them. For example in the case of the $X$ (3872) meson, $j_{H_{1}^{0}}$ corresponds to the $j_{\mu}^{(2)}$ current, and $j_{H_{2}^{0}}$ corresponds to the $j_{\mu}^{(4)}$ current appearing in Eq. (1). For a determination of the mixing angles we consider a correlator which is formed from two orthogonal currents corresponding to two different hadronic states,

$\Pi=i \int \mathrm{d}^{4} x \mathrm{e}^{i p x}\left\langle 0\left|T\left\{j_{H_{1}}(x) \bar{j}_{H_{2}}(0)\right\}\right| 0\right\rangle$.

In the present work we calculate the mixing angles among the two-quark and four-quark states given by Eqs. (1), (4), and (5). The origin of this mixing can be explained as follows: The $\bar{c} c$ state can emit a gluon, which subsequently splits into a light quark-antiquark living like a molecular state during some time interval. According to the general strategy of the QCD sum rules method, this correlation function is calculated in terms of hadrons on the physical side, and in terms of quarks and gluons on the theoretical side. Using the duality ansatz these two representations are then equated to obtain the QCD sum rules. The correlation function from the hadronic side is calculated by saturating it with the corresponding hadrons carrying the same quantum numbers as the interpolating current. Obviously, the hadronic part of the correlation function should be equal to zero after this procedure, since the hadronic states given by Eq. (8) are orthogonal. Using Eq. (8) in the theoretical calculation of the correlation function we get

$\Pi=\sin \theta \cos \theta\left[\Pi_{22}^{(0)}-\Pi_{11}^{(0)}\right]+\left(\cos ^{2} \theta-\sin ^{2} \theta\right) \Pi_{12}^{(0)}=0$,

where $\Pi_{i j}^{(0)}$ is the correlation function corresponding to the unmixed case, i.e.,

$\Pi_{i j}^{(0)}=i \int \mathrm{d}^{4} x \mathrm{e}^{i p x}\left\langle 0\left|T\left\{j_{H_{i}}^{(0)}(x) \bar{j}_{H_{j}}^{(0)}(0)\right\}\right| 0\right\rangle$,

where $i=1$ or 2 , and $j=1$ or 2 .

In the case of a scalar current, the correlation function contains only one invariant function, which we shall denote by $\Pi_{i j}^{(0)}$. In the case of a vector (axial-vector) current the two-point correlation function can be written in terms of two independent invariant functions as follows:

$\Pi_{\mu \nu i j}^{(0)}\left(p^{2}\right)=\Pi_{i j}^{(1)}\left(p^{2}\right)\left(g_{\mu \nu}-\frac{p_{\mu} p_{\nu}}{p^{2}}\right)+\Pi_{i j}^{(2)}\left(p^{2}\right) \frac{p_{\mu} p_{v}}{p^{2}}$.

From Eq. (10), for the mixing angle we get

$\tan 2 \theta=\frac{2 \Pi_{12}^{(0)}}{\Pi_{11}^{(0)}-\Pi_{22}^{(0)}}$.

The invariant functions $\Pi_{i j}^{(1)}\left(p^{2}\right)$ and $\Pi_{i j}^{(2)}\left(p^{2}\right)$ in Eq. (11) are associated with the spin-1 and spin-0 mesons, respectively. Since $X(3872)$ and $Y(4260)$ mesons both have the quantum number $J=1$, in the further discussions we shall consider only the structure $\left(g_{\mu \nu}-p_{\mu} p_{\nu} / p^{2}\right)$, i.e., we shall analyze the invariant function $\Pi_{i j}^{(1)}\left(p^{2}\right)$ only.

Using the currents given in Eqs. (1), (4), (7), and their respective orthogonal combinations, the corresponding correlation functions are calculated in terms of quarks and gluons in the deep Euclidean region $p^{2} \ll 0$ using the operator product expansion [19-22]. The results for each current are presented in the Appendix.

Having all the necessary formulas, we can now proceed to the numerical analysis of the mixing angles. It follows from the expressions of the mixing angles that the main input parameters involved in the numerical calculations are the 
quark and gluon condensates and the masses of the quarks whose values are given as $\langle\bar{q} q\rangle(1 \mathrm{GeV})=-\left(246_{-19}^{+28} \mathrm{MeV}\right)^{3}$ [23] (as regards the values of the input parameters see also [24-38], $\left\langle g_{s}^{2} G^{2}\right\rangle=0.47 \mathrm{GeV}^{4}, m_{0}^{2}=0.8 \mathrm{GeV}^{2}$ [39]. For the mass of the $c$ quark we have used its $\overline{M S}$ value $\bar{m}_{c}\left(\bar{m}_{c}\right)=$ $1.28 \pm 0.03 \mathrm{GeV}[40]$.

Beside these input parameters, the sum rules do also contain two auxiliary parameters, namely, the Borel mass parameter $M^{2}$ and the continuum threshold $s_{0}$. The continuum threshold is correlated with the energy of the first excited state. It is usually chosen as $\sqrt{s_{0}}=\left(m_{\text {ground }}+0.5\right) \mathrm{GeV}$ and we look for the domain of $s_{0}$ which satisfies this restriction. In this work the continuum thresholds for $X$ (3872), $Y$ (3940) and $Y(4260)$ are chosen as $\sqrt{s_{0}}=(4.2 \pm 0.1) \mathrm{GeV}$ [9], $\sqrt{s_{0}}=(4.4 \pm 0.1) \mathrm{GeV}[11,12], \sqrt{s_{0}}=(4.7 \pm 0.1) \mathrm{GeV}$ [14], respectively, which are obtained from the analysis of two-point function. The working region of the Borel mass parameter $M^{2}$ can be obtained using the following procedure. The upper bound of $M^{2}$ is determined from the condition that the continuum and higher state contributions constitute about $30 \%$ of the pole contribution, i.e.,

$\frac{\int_{s_{0}}^{\infty} \mathrm{d} s \rho(s) \mathrm{e}^{-s / M^{2}}}{\int_{4 m_{c}^{2}}^{\infty} \mathrm{d} s \rho(s) \mathrm{e}^{-s / M^{2}}}<1 / 3$,

where $\rho(s)$ is the spectral density which is related to the imaginary part of the invariant function $\Pi(s)$ according to

$\rho(s)=\frac{1}{\pi} \operatorname{Im} \Pi(s)$.

The lower bound for $M^{2}$ is determined from the condition that the perturbative contributions exceed the nonperturbative ones. From these conditions we get the following working regions for $M^{2}: 2 \leq M^{2} \leq 4 \mathrm{GeV}^{2}$ for $X(3872), Y(3940)$, and $2 \leq M^{2} \leq \overline{5} \mathrm{GeV}^{2}$ for the $g_{\mu \nu}-p_{\mu} p_{\nu} / p^{2}$ structure.

In Fig. 1 we present the dependence of the mixing angle $\theta$ on the Borel mass parameter $M^{2}$ at $\sqrt{s_{0}}=(4.4 \pm 0.1) \mathrm{GeV}$ for the $g_{\mu \nu}-p_{\mu} p_{\nu} / p^{2}$ structure for the $X$ (3872) state. We observe from this figure that this structure predicts for the mixing angle $\theta \simeq(2.4 \pm 0.6)^{0}$, which is considerably small compared to the value of $\theta=(9 \pm 4)^{0}$ obtained in [9] in order to reproduce the mass of $X(3872)$.

In Fig. 2 we present the dependence of the mixing angle $\theta$ on $M^{2}$ at a fixed value of $\sqrt{s_{0}}=(4.4 \pm 0.1) \mathrm{GeV}$ for the $Y(3940)$ state. The mixing angle we obtain from this figure is $\theta=(20 \pm 2)^{0}$, which is about 3.5 times smaller compared to the mixing angle $\theta=(76 \pm 5)^{0}$, which is predicted in $[11,12]$.

In Fig. 3 we present the dependence of the mixing angle $\theta$ on the Borel mass parameter $M^{2}$ at $\sqrt{s_{0}}=(4.6 \pm 0.1) \mathrm{GeV}$ for the $g_{\mu \nu}-p_{\mu} p_{\nu} / p^{2}$ structure for the $Y(4260)$ state. We see from this figure that the mixing angle has the value $\theta=$

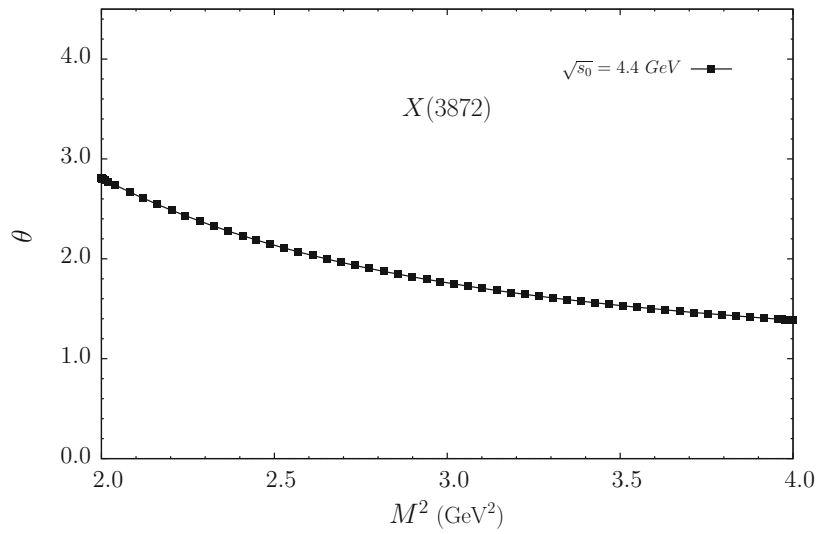

Fig. 1 The dependence of the mixing angle $\theta$ on the Borel mass square $M^{2}$, at the fixed value of the continuum threshold $\sqrt{s_{0}}=4.4 \mathrm{GeV}$, for the structure $g_{\mu \nu}-p_{\mu} p_{\nu} / p^{2}$ for the $X(3872)$ state

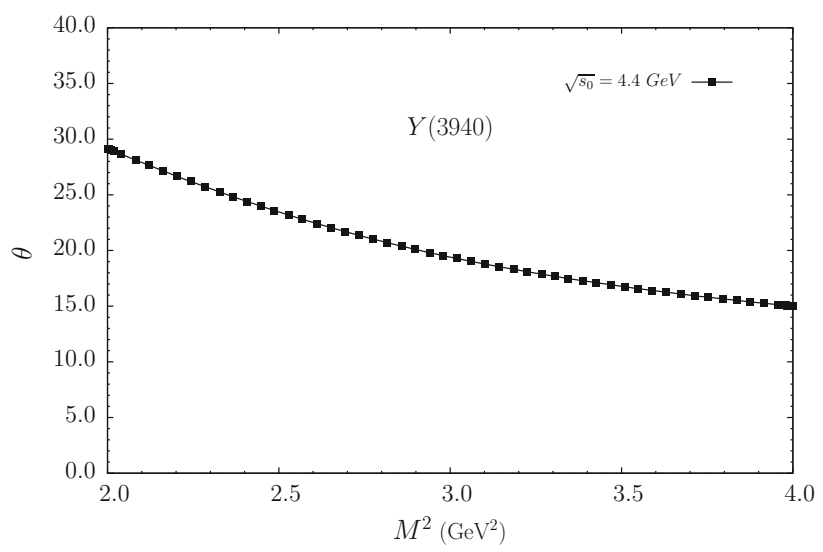

Fig. 2 The dependence of the mixing angle $\theta$ on the Borel mass square $M^{2}$, at the fixed value of the continuum threshold $\sqrt{s_{0}}=4.4 \mathrm{GeV}$, for the $Y$ (3940) state

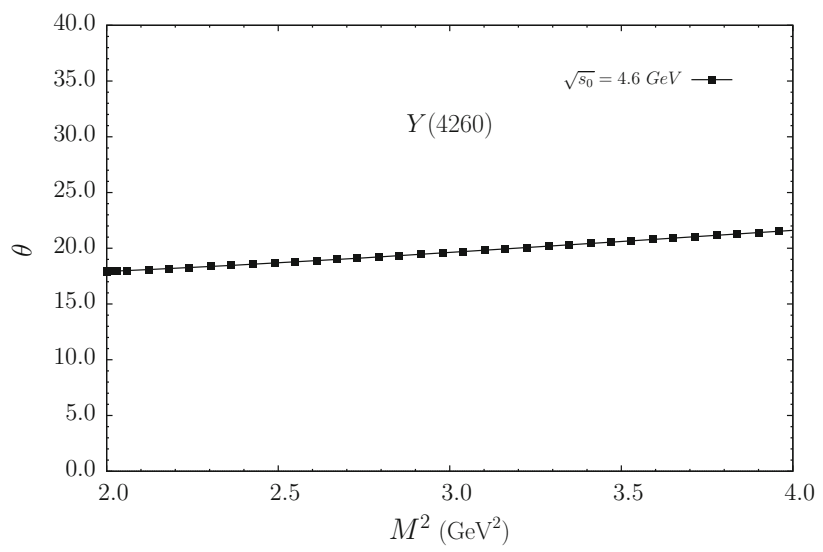

Fig. 3 The same as in Fig. 1, but at the fixed value of the continuum threshold $\sqrt{s_{0}}=4.6 \mathrm{GeV}$ for the $Y(4260)$ state

$(20 \pm 3)^{0}$, which is approximately 2.5 times smaller than the mixing angle $\theta=(53 \pm 0.5)^{0}$, predicted in [14]. We finally note that, using the mixing angles obtained in this work, the 
masses of the states orthogonal to the $X(3872), Y(3940)$, and $Y(4260)$ are calculated in [41].

In summary, in this work, based on the assumptions that the $X(3872)$ is a mixture of charmonium and $D^{* 0} \bar{D}^{0}$ states, $Y(3940)$ is a mixture of scalar $\bar{c} c$ and $D^{*} D^{*}$ molecule, and $Y(4260)$ is a mixture of tetra-quark and charmonium states, we estimate the respective mixing angles within the QCD sum rules method. The result is obtained that the mixing angles calculated in the present work for all considered pictures are considerably smaller than the ones predicted in $[9,11,12,14]$. Therefore, in our view the considered pictures for $X(3872), Y(3940)$, and $Y(4260)$ are not successful in describing these states.

Open Access This article is distributed under the terms of the Creative Commons Attribution 4.0 International License (http://creativecomm ons.org/licenses/by/4.0/), which permits unrestricted use, distribution, and reproduction in any medium, provided you give appropriate credit to the original author(s) and the source, provide a link to the Creative Commons license, and indicate if changes were made.

Funded by SCOAP ${ }^{3}$.

\section{Appendix A}

In this appendix we present the spectral densities for the $Y(4260), X(3872)$, and $Y(3940)$ states.

\section{$\mathbf{Y}(4260)$}

Spectral densities corresponding to the $g_{\mu \nu}-p_{\mu} p_{\nu} / p^{2}$ structure:

$$
\begin{aligned}
& \rho_{11}(s)-\rho_{22}(s)=-\frac{1}{3072 \pi^{6}} \int_{\alpha_{\min }}^{\alpha_{\max }} \mathrm{d} \alpha \int_{\beta_{\min }}^{\beta_{\max }} \mathrm{d} \beta \\
& \times\left\{12(1-\alpha-\beta) \mu_{1}^{2} m_{q}^{2}\left(\alpha \beta \mu_{1}+6 m_{Q^{m}} m_{Q^{\prime}}\right)\right. \\
& -\mu_{1}^{3}(1-\alpha-\beta)\left[3 \alpha \beta(1+\alpha+\beta) \mu_{1}\right. \\
& \left.-2(1-\alpha-\beta)^{2} m_{Q^{m}} m_{Q^{\prime}}\right] \\
& +16 \pi^{2} m_{q}\left[12 \alpha \beta \mu_{1}^{2}+m_{0}^{2} m_{Q^{m}}{ }_{Q^{\prime}}\right. \\
& \left.\left.+6(5-\alpha-\beta) \mu_{1} m_{Q} m_{Q^{\prime}}\right]\langle\bar{q} q\rangle\right\} \\
& +\frac{\langle\bar{q} q\rangle}{192 \pi^{4}} \int_{\alpha_{\min }}^{\alpha_{\max }} \mathrm{d} \alpha\left\{m _ { q } ( 1 - \alpha ) \alpha \left[6 \mu_{2}^{2}\right.\right. \\
& \left.-m_{0}^{2}\left(\mu_{2}+2 s\right)\right]-6 m_{0}^{2} m_{q} m_{Q} m_{Q^{\prime}} \\
& -8 \pi^{2}\left[(1-\alpha) \alpha\left(m_{0}^{2}-7 \mu_{2}+8 s-2 m_{q}^{2}\right)\right. \\
& \left.\left.+10 m_{Q^{m}}{ }_{Q^{\prime}}\right]\right\} \text {, } \\
& \rho_{12}(s)=\rho_{21}(s)=-\frac{\langle\bar{q} q\rangle^{2}}{8 \pi^{2}} \int_{\alpha_{\min }}^{\alpha_{\max }} \mathrm{d} \alpha[(1-\alpha) \\
& \left.\times \alpha\left(\mu_{2}-s\right)-m_{Q^{m}}{ }_{Q^{\prime}}\right] .
\end{aligned}
$$

\section{$\mathbf{X}(3872)$}

Spectral densities corresponding to the $g_{\mu \nu}-p_{\mu} p_{\nu} / p^{2}$ structure:

$$
\begin{aligned}
& \rho_{11}(s)-\rho_{22}(s)=\frac{3 \mu_{1}}{4096 \pi^{6}} \int_{\alpha_{\min }}^{\alpha_{\max }} \mathrm{d} \alpha \int_{\beta_{\min }}^{\beta_{\max }} \mathrm{d} \beta\{-\alpha \beta \\
& \times\left[1-(\alpha+\beta)^{2}\right] \mu_{1}^{3}+2(1-\alpha-\beta) \mu_{1} m_{q}[\alpha(3+\alpha+\beta) \\
& \left.\times \mu_{1} m_{Q^{\prime}}+\beta(3+\alpha+\beta) \mu_{1} m_{Q}-12 m_{q} m_{Q^{2}} m_{Q^{\prime}}\right] \\
& -8 \pi^{2}\langle\bar{q} q\rangle\left[m_{0}^{2}-2(1+\alpha+\beta) \mu_{1}+2 m_{q}^{2}\right] \\
& \left.\times\left(\alpha m_{Q^{\prime}}+\beta m_{Q}\right)+32 \pi^{2} m_{q}\langle\bar{q} q\rangle\left(\alpha \beta \mu_{1}-4 m_{Q m_{Q^{\prime}}}\right)\right\} \\
& +\frac{\langle\bar{q} q\rangle}{512 \pi^{4}} \int_{\alpha_{\min }}^{\alpha_{\max }} \mathrm{d} \alpha\left\{6 m_{0}^{2} \mu_{2}\left[(1-\alpha) m_{Q}+\alpha m_{Q^{\prime}}\right]\right. \\
& -32 \pi^{2}\left[m_{Q^{m}}{ }_{Q^{\prime}}+m_{q}^{2} \alpha(1-\alpha)\right]\langle\bar{q} q\rangle \\
& +3 m_{q}^{2}\left(m_{0}^{2}+4 \mu_{2}\right)\left[(1-\alpha) m_{Q}+\alpha m_{Q^{\prime}}\right] \\
& -4 m_{q} \alpha(1-\alpha)\left[3 \mu_{2}^{2}+m_{0}^{2}\left(\mu_{2}-s\right)\right]-12 m_{0}^{2} m_{q} m_{Q} m_{Q^{\prime}} \\
& \left.+24 \pi^{2} m_{q}\left[(1-\alpha) m_{Q}+\alpha m_{Q^{\prime}}\right]\langle\bar{q} q\rangle\right\} \text {, } \\
& \rho_{12}(s)=\rho_{21}(s)=-\frac{\langle\bar{q} q\rangle^{2}}{96 \pi^{2}} \int_{\alpha_{\min }}^{\alpha_{\max }} \mathrm{d} \alpha \\
& \times\left[\alpha(1-\alpha)\left(\mu_{2}-s\right)+m_{Q^{m}}{ }_{Q^{\prime}}\right] .
\end{aligned}
$$

\section{Y(3940)}

Spectral densities for the $Y(3940)$ state:

$$
\begin{aligned}
& \rho_{11}(s)-\rho_{22}(s)=\frac{3 \mu_{1}}{512 \pi^{6}} \int_{\alpha_{\min }}^{\alpha_{\max }} \mathrm{d} \alpha \int_{\beta_{\min }}^{\beta_{\max }} \mathrm{d} \beta\{-(1-\alpha-\beta) \\
& \times \mu_{1}\left[\alpha \beta \mu_{1}^{2}+12 m_{q}^{2} m_{Q^{m}} m_{Q^{\prime}}-2 \mu_{1} m_{q}\left(\alpha m_{Q^{\prime}}+\beta m_{Q}\right)\right] \\
& \left.+8 \pi^{2}\left[\mu_{1}\left(\alpha m_{Q^{\prime}}+\beta m_{Q}\right)-8 m_{q} m_{Q^{m}} m_{Q^{\prime}}\right]\langle\bar{q} q\rangle\right\} \\
& +\frac{\langle\bar{q} q\rangle}{384 \pi^{4}} \int_{\alpha_{\min }}^{\alpha_{\max }} \mathrm{d} \alpha\left\{6\left(m_{0}^{2}+3 \mu_{2}\right) m_{q}^{2}\left[(1-\alpha) m_{Q}+\alpha m_{Q^{\prime}}\right]\right. \\
& +9 m_{0}^{2} \mu_{2}\left[(1-\alpha) m_{Q}+\alpha m_{Q^{\prime}}\right] \\
& -12 m_{q} \alpha(1-\alpha)\left[3 \mu_{2}^{2}+m_{0}^{2}\left(2 \mu_{2}-s\right)\right]-36 m_{0}^{2} m_{q} m_{Q^{m} m_{Q^{\prime}}} \\
& +16 \pi^{2}\left[-\alpha(1-\alpha)\left(14 \mu_{2}-7 s++9 m_{q}^{2}\right)+3(1-\alpha) m_{q} m_{Q}\right. \\
& \left.\left.+\left(3 \alpha m_{q}-13 m_{Q}\right) m_{Q^{\prime}}\right]\langle\bar{q} q\rangle\right\}, \\
& \rho_{12}(s)=\rho_{21}(s)=\frac{\langle\bar{q} q\rangle^{2}}{4 \sqrt{2} \pi^{2}} \int_{\alpha_{\min }}^{\alpha_{\max }} \mathrm{d} \alpha \\
& \quad \times\left[\alpha(1-\alpha)\left(2 \mu_{2}-s\right)+m_{\left.Q^{m} m_{Q^{\prime}}\right],}\right.
\end{aligned}
$$


where

$$
\begin{aligned}
\mu_{1} & =\frac{m_{Q}^{2}}{\alpha}+\frac{m_{Q^{\prime}}^{2}}{\beta}-s, \\
\mu_{2} & =\mu(\beta \rightarrow 1-\alpha), \\
\beta_{\min } & =\frac{\alpha m_{Q^{\prime}}^{2}}{s \alpha-m_{Q}^{2}}, \\
\beta_{\max } & =1-\alpha, \\
\alpha_{\min } & =\frac{1}{2 s}\left[s+m_{Q}^{2}-m_{Q^{\prime}}^{2}-\sqrt{\left(s+m_{Q}^{2}-m_{Q^{\prime}}^{2}\right)^{2}-4 m_{Q^{s}}^{2}}\right], \\
\alpha_{\max } & =\frac{1}{2 s}\left[s+m_{Q}^{2}-m_{Q^{\prime}}^{2}+\sqrt{\left(s+m_{Q}^{2}-m_{Q^{\prime}}^{2}\right)^{2}-4 m_{Q^{s}}^{2}}\right] .
\end{aligned}
$$

\section{References}

1. S.K. Choi et al., Phys. Rev. Lett. 91, 262001 (2003)

2. E. Swanson, Phys. Rep. 429, 243 (2006)

3. E. Klempt, A. Zaitsev, Phys. Rep. 454, 1 (2007)

4. S. Godfrey, S. Olsen, Ann. Rev. Nucl. Part. Sci. 58, 51 (2008)

5. M. Nielsen, F.S. Navarra, S.H. Lee, Phys. Rep. 497, 41 (2010)

6. N. Brambilla et al., Eur. Phys. J. C 71, 1534 (2011)

7. A. Esposito, A.L. Guerrieri, P. Piccinini, A. Pilloni, A.D. Polosa, Int. Mod. Phys. A 30, 1530002 (2014). arXiv:1411.5997 [hep-ph]

8. M.A. Shifman, A.I. Vainshtein, V.I. Zakharov, Nucl. Phys. B 147, 385 (1979)

9. R.D. Matheus, F.S. Navarra, M. Nielsen, C.M. Zanetti, Phys. Rev. D 80, 056002 (2009)

10. Z.G. Wang, T. Huang, Phys. Rev. D 89, 054019 (2014)

11. R.M. Albuquerque, J.M. Dias, M. Nielsen, C.M. Zanetti, Phys. Lett. B 702, 359 (2011)

12. R.M. Albuquerque, J.M. Dias, M. Nielsen, C.M. Zanetti, Phys. Rev. D 89, 076007 (2014)

13. Z.G. Wang, Eur. Phys. J. C 74, 2963 (2014)

14. J.M. Dias, R.M. Albuquerque, M. Nielsen, C.M. Zanetti, Phys. Rev. D 86, 116012 (2012)
15. M. Takizawa, K. Tsushima, Y. Kohyama, K. Kubodera, Prog. Theor. Phys. 82, 481 (1989)

16. T. Feldmann, P. Kroll, B. Stech, Phys. Rev. D 58, 114006 (1998)

17. T.M. Aliev, A. Özpineci, V.I. Zamiralov, Phys. Rev. D 83, 016008 (2011)

18. T.M. Aliev, K. Azizi, M. Savc1, Phys. Lett. B 715, 149 (2012)

19. K. Wilson, Phys. Rev. 179, 1499 (1969)

20. K. Wilson, Phys. Rev. D 3, 1818 (1971)

21. K.G. Chetyrkin, S.G. Gorishny, F.V. Tkachov, Phys. Lett. B 119, 407 (1983)

22. K.G. Chetyrkin, Phys. Lett. B 126, 371 (1983)

23. B.L. Ioffe, Prog. Part. Nucl. Phys. 56, 232 (2006)

24. S. Narison, Camb. Monogr. Part. Phys. Nucl. Phys. Cosmol. 17, 1 (2002)

25. S. Narison, World Sci. Lect. Notes Phys. 26, 1 (1989)

26. S. Narison, Acta Phys. Polon. B 26, 687 (1995)

27. S. Narison, Riv. Nuovo Cimento Soc. Ital. Fis. 10, 1 (1987)

28. S. Narison, Phys. Rep. 84, 263 (1982)

29. S. Narison, Phys. Lett. B 624, 223 (2005)

30. S. Narison, Phys. Lett. B 466, 349 (1999)

31. S. Narison, Phys. Lett. B 387, 162 (1996)

32. H. Forkel, M. Nielsen, Phys. Lett. B 345, 55 (1995)

33. R.M. Albuquerque, S. Narison, M. Nielsen, Phys. Lett. B 684, 236 (2010)

34. R.M. Albuquerque, S. Narison, Phys. Lett. B 694, 217 (2010)

35. M. Eidemuller, F.S. Navarra, M. Nielsen, R.R. Silva, Phys. Rev. D 72, 034003 (2005)

36. R.D. Matheus, F.S. Navarra, M. Nielsen, R.R. Silva, Phys. Lett. B 578, 323 (2004)

37. R.D. Matheus, F.S. Navarra, M. Nielsen, R.R. Silva, Phys. Lett. B 602, 185 (2004)

38. R.M. Albuquerque, F. Fanomezana, S. Narison, A. Rabemananjara, Phys. Lett. B 715, 129 (2012)

39. V.M. Belyaev, B.L. Ioffe, Sov. Phys. JETP 57, 716 (1983)

40. K.G. Chetrykin et al., Phys. Rev. D 80, 074010 (2009)

41. T.M. Aliev, H. Özşahin, M. Savcı, Adv. High Energy Phys. 2015, 728098 (2015) 